\title{
المسلمون وكتابة التاريخ
}

\section{المؤلف: الدكتور عبد العليم عبد الرحمن خضر \\ الناشر : المعهد العالمي للفكر الإسلامي (هيرندن-فيرجينا)}

الدار العالمية الكتاب الإسلامي (الرياض)، سلسة المنهجية الإسلامية (6) الطبعة الثانية:

1415هـ 1995م، (319ص) (195) (19)

تقديم

يعالج هذا الكتاب العديد من القضايا المتصلة بنشأة علم التاريخ، وتطوره، وعلاقته بالعلوم الأخرى، مُركزاً بشكل خاص على المسائل المتعلقة بمنهج البحث التاريخي، وفلسفة التاريخ، والقوانين والسنن التي تحكم حركة التاريخ. والناظر في الكتاب يلحظ بشكل جلي الجهد الذي بذله المؤلف في تجلية القضايا التي تناولما، وهي بدون شك قضايا شائكة ما زالت مثار نقاش وأخذ وردّ بين المشتغلين بالدراسات التاريخية.

لقد انطلق المؤلف في معالجته لموضوعاته من تصور إسلامي واضح؛ لذا يحسب للمؤلف هذا الجههد في خدمة مشروع إسلامية علم التاريخ. وبعبارة أخرى، فإن هذا الكتاب يحمل في طياته دعوة مخلصة للمشتغلين بالدراسات التاريخية ليوحد جهودهم ويوجهوا طاقاتم لخدمة منهج البحث التاريخي الإسلامي؛ ليسد هذا المنهج ثغرة في خدمة مشروعنا الحضاري المعاصر.

إن الهدف الرئيس من تأليف هذا الكتاب كما يقول المؤلف: "إثبات أن (التاريخ) ليس دخيلاً على التفكير الإسلامي ولم يقتبسه المسلمون من غيرهم، بل إن مناهج البحث التاريخي التي عرفتها (أوروبا) إنما اعتمدت بالدرجة الأولى على جهود علماء المسلمين في هذا المجال... والحقيقة التي أرمي إلى بياها هي ضرورة العودة إلى منهج إسلامي لكتابة التاريخ بعد أن انتشرت في عالمنا المعاصر مناهج منحرفة شوهت قضية الفكر التاريخي في حضارة المسلمين". 
ونخن مع المؤلف في دعوته تلك، فالحضارة الإسلامية حضارة مناهج، ففي كل علم من العلوم

الإسلامية نجد أصول منهج: أصول الفقه، أصول الحديث (علم مصطلح الحديث)، أصول التفسير ... إلخ. وكل هذه العلوم تلتقي عند الأصول الثابتة للمنهج ثم تفترق وتتمايز في دوائر لكل منها منهجه الخاص، ولكن هذه المناهج ما زالت بحاجة إلى مراجعة وإعادة نظر تتخللها عمليات تفكيك وتحليل، ثم إعادة تركيب وصياغة لإعادة الحيوية إليها؛ لتكون قادرة على مواكبة الثورة المائلة في حقل المناهج، وقادرة على التعايش معها في ظل فلسفة الأخذ والعطاء المتركزة على ضوابط منهجية صارمة.

لقد أثمرت جهود الغرب المكثفة منذ أكثر من خمسة قرون لاكتشاف المنهج الأصلح للتعامل مع التاريخ العديد من النظريات والدراسات التي ترتكز على منطق منهجي واضح، وهي بحق جهود تستحق

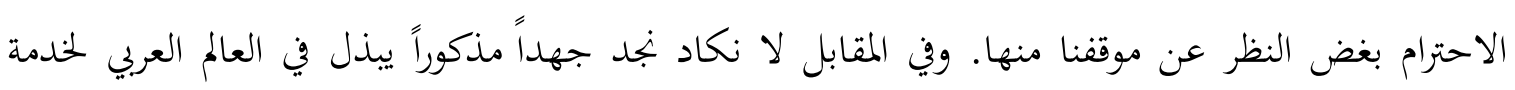
هذه الغاية. وما لبثت النظريات الغربية المسلحة بقوة الطرح أن فرضت هيمنتها على قطاع لا بأس به من المشتغلين بالدراسات التاريخية في عالمنا الإسلامي مما ترتب عليه هذه الصورة التي نراها في العديد من الكتابات التي اتخذت من الطعن في ثوابت الأمة الحضارية، والتاريخية منهجاً لها متذرعة بالموضوعية والمنهجية التي تم استيرادها من بيئات لها خصائصها الحضارية والمتركزة على تصورات خاصة بتلك البيئات والتي لا تصلح في معظمها لتفسير حركة تاريخنا وفهم كنه حضارتنا الإسلامية. وهذا الإشكال كان ضمن الدوافع الرئيسة -كما تقدم- التي دفعت الدكتور عبد العليم خضر لتأليف كتابه هذا.

\section{مضمون الكتاب والقضايا الرئيسة فيه}

وقد جاء كتاب المسلمون وكتابة التاريخ في سبعة فصول مسبوقة بمقدمة. ومن أهم القضايا التي تناولها في الفصل الأول، (المفهوم العلمي للتاريخ وعلاقته بالعلوم الاجتماعية)، فبعد أن بيّن المدلول الحقيقي للتاريخ والوظيفة الأساسية له، ومكانته المرموقة في فهم معطيات العلاقات الدولية تفسيراً وتحليلاً، نجده يؤكد أن التاريخ السياسي لا يمثل التاريخ الحقيقي للشعوب ولا يمكن استخلاص القوانين والسنن منه، إنا التاريخ الحقيقي هو الذي يرصد حركة الإنسان في بجالاتما الاجتماعية والاقتصادية؛ لذا كانت علاقة علم التاريخ بالعلوم الأخرى علاقة وطيدة. 
وتحدث المؤلف في هذا الفصل أيضاً، بعد تناوله لموضوع منهج البحث التاريخي والمراحل التي يمر بها الباحث، عن المناهج المتطورة في حقل الدراسات التاريخية التي ظهرت في السنوات الأخيرة، وخاصة ما يعرف بالمنهج الكمي الذي يساعد على التوصل إلى مؤشرات معينة من خلال الإحصاء والبيانات والجداول. وخلص المؤلف في غاية الفصل إلى أن أهمية علم التاريخ تكمن في أخذ العظة والاعتبار، وتجنب الوقوع في الأخطاء.

وأفرد المؤلف الفصل الثاني لعلم التاريخ عند المسلمين، مؤكداً أن عملية التدوين التاريخي نشأت مستقلة في موضوعاتا وتقيمها الخاص عن تاريخ الأمم الأخرى، وبالتالي لم يكن التاريخ الإسلامي استمراراً أو صلة للتواريخ القديمة، وإنما هو تاريخ إسلامي خالص أسهمت عدة عوامل في تطوره وازدهاره.

وتتبع المؤلف في هذا الفصل مسيرة التأليف التاريخي بشكل مختصر مشيراً إلى بعض مناهج كبار المؤرِخين، وخلص في غاية هذا التتبع إلى اعتبار تاريخ اليعقوبي وتاريخ الأمم والملوك، ومروج الذهب، نماذج للتاريخ الإسلامي العالمي في ذلك الحين، أما التاريخ المحلي فعدّه المؤلف تعبيراً حياً عن التنوع والوحدة في المجتمعات الإسلامية.

وأكد المؤلف في الفصل الثالث الذي خصصه لمسألة الحياد والصدق في تدوين الحدث التاريخي نزاهة المؤرخ المسلم، موضحاً أن السرّ في سلامة الفكر التاريخي في الحضارة الإسلامية هو عدم اتباع الظن والابتعاد عن هوى النفس من جهة، والتشدد في إحراز العلم أو اليقين من جهة أخرى. فواجب المؤرخ العمل على الكشف بأمانة عن القوانين الصحيحة التي توجه بجرى التاريخ، ومن ثَُّّ عليه أن يتحرى الدقة ما استطاع إلى ذلك سبيلاً، وأن ينظر إلى القضية نظرة شمولية بعيداً عن أية مؤثرات، خاصة في ظل انتشار ظاهرة الوضع والكذب في الأخبار التي تعود حسب رأي ابن خلدون إلى عدة أسباب منها:

1- التشيع للآراء والمذاهب. 2- الثقة بالناقلين. 3- الذهول عن المقاصد. 4- توهم الصدق. 5الجهل بطبائع الأحوال في العمران. 6- تقرب الناس لأصحاب السلطان وأهل الرتب. 7- الجهل بتطبيق الأحوال على الوقائع. 8- الغفلة وعدم الإحاطة بما يلزم المؤرخ من العلوم والمعارف التي تساعده على تقصي 
لذا نجد ابن خلدون يرفض العديد من القصص والروايات في كتب التفسير والسير والتاريخ لتهافتها أمام المنهج الذي وضعه لنقد الروايات. ومن هذه الروايات والقصص: قصة إرَّ ذات العماد، وبناء

$$
\text { الإسكندرية، ونكبة البرامكة... إلخ. }
$$

وجاء الفصل الرابع ليعالج ملامح المنهج العلمي لكتابة التاريخ عند ابن خلدون. وفيه يؤكد المؤلف أن ابن خلدون هو أبو علم التاريخ، أو مؤسس علم التاريخ على حد تعبير الدكتور آلبان ج. ويدجري. فالتاريخ غدا على يد ابن خلدون يحكمه منهج محدد المعالم يقوم على الملاحظة والمقارنة ودراسة البيئة بمفهومها الشامل. هكذا أصبح التاريخ عنده علماً يرتبط بالعلوم الأخرى بشبكة من العلاقات التي لا يمكن للمؤرخ تجاهلها؛ لذا نجده يشترط في المؤرخ أن يتسلح بمعارف متنوعة فضلاً عن حسن النظر والتثبت. كما يهتم ابن خلدون بفكرة التطور التاريخي في الحياة الاجتماعية، فقد ركز على العلاقات الاجتماعية وأثرها في تطوير أنماط العيش عند الشعوب عبر التاريخ، فتحدث عن العصبية بأنواعها، مؤكداً أثر الأخلاق في قيام الممالك وسقوطها، وهكذا نقل ابن خلدون الحادثة التاريخية من إطارها الجزئي إلى إطارها الكلي، فغدا علم التاريخ على يديه علماً متكاملاً بأسسه ومناهجه.

ويختم المؤلف هذا الفصل بالحديث عن صورة الكتابة التاريخية قبل ابن خلدون من خلال استعراضه لمجموعة من المؤرخين الكبار أمثال: ابن عبد الحكم، والكندي، وابن زولاق والنويري... مشيراً إلى أسلوب السجع الذي ابتليت به الكتابات التاريخية منذ مرحلة متقدمة خاصة في كتب تراجم الحكام، وأن سيد هذا الفن هو العماد الأصفهاني الذي كتب جميع مؤلفاته التاريخية بهذا الأسلوب.

وخصص المؤلف الفصل الخامس لمناهج البحث التاريخي عند المسلمين، مبيّناً أن المؤرِّين المسلمين لم يتركوا فرعاً من فروع التاريخ إلا وكتبوا فيه، فقد كتبوا في: السير، والطبقات، وأخبار النساء، والخطط والأمصار، والتاريخ العام... إلخا، ومشيراً إلى أشهر من ألّف في هذه الحقول مع الإلماح بشكل عام للمنهجية المتبعة في التدوين. وقد أوضح الدكتور خضر أن الخبر التاريخي رغم اختلاف بيانه بقي محتفظاً بخصائص القصة القصيرة والمروية بشكل حسّي مع إبراز الوقائع المثيرة، وأنّ المدرسة الإخبارية تأثرت بأسلوب المحدّثين 
بشكل واضح أخذت تستشهد بالشعر وتستخدم الوثائق في مروياتا، لا بل إفم استخدموا النقوش والأختام إلى جانب المعاينة بوصفها مصادر لمروياتم.

وانتهى الأمر بالحركة التاريخية إلى ظهور منهج جديد في تدوين الأحداث التاريخية وهو المنهج الحولي، الذي يعد الكندي من أشهر الممثلين له من خلال حرصه على تتبع الخبر من منابعه الأولى وحرصه على تسجيل الحوادث باليوم والشهر والسنة. أما الدينوري صاحب الأخبار الطوال، فقد راعى التسلسل الزمني مع انتقاء الأخبار وفقاً لمفهوم التاريخ الإسلامي العالمي مع التوسع في الحوادث والحركات. ثم تناول المؤلف كورت منهج السخاويّ في التدوين التاريخي من خلال كتابه الإعلان بالتوبيخ لمن ذم التاريخ؛ فقد انبرى السخاوي مدافعاً عن هذا العلم الذي عرفه بأنه تعريف بالوقت، وبيّن أن فائدته تنحصر في معرفة الأمور على وجهها، أما غايته: فالرجاء لرضا الله. وقد ذكر السخاوي مجموعة من الشروط الواجب توافرها في المؤرخ ومنها:

1- العدالة مع الضبط التام الناشئ عنه مزيد الإتقان. 2- أن يكون عالماً بطرق النقل. 3- أن يكون عارفاً بمقادير الناس وبأحوالهم ومنازهم. 4- وأن يكون ذا قدرة على التمييز بين المقبول والمردود. 5وأن يكون متصفاً بالورع والتقوى، بعيداً عن الظن السيء. ويختم المؤلف هذا الفصل بالحديث بشكل مختصر عن نظرية علم التاريخ عند الكافيجي. وعقد المؤلف الفصل السادس للاتحاهات الحديثة في بجال البحث التاريخي، فتحدث عن نظرية التفسير المادي للتاريخ، التي عدّت الدين والإيمان مجرد نتاج أو انعكاس للأوضاع المادية والعلاقات الاجتماعية. كما تحدث عن وليم ونورد ريد (1875-1838) صاحب النظرية المتطرفة عن المذهب الطبيعي في تفسير التاريخ، الذي وضع في هذا الكتاب أفكاره الشاذة عن (الطبعانية) في بحث بعنوان "استشهاد الإنسان" خلص فيها إلى أن الإنسان عبارة عن ثمرة لعمليات الطبيعة.

وتحدث المؤلف في هذا الفصل أيضاً عن الثقافة الذاتية وتفسير التاريخ وتقاربه مع العلوم الأخرى مثل علم الإنسان، والأحياء، والحساب. ثم يختم المؤلف الفصل بالحديث عن أحدث الاتحاهات إلى ظهرت في 
السنوات الأخيرة، والتي ترى أن من واجب أن يعد منهاجاً أو إطاراً للتكهن. فالتاريخ يجب أن يهتم دائماً بالمستقبل، ولو أن هذا المستقبل قد يكون وثيق الصلة بماض بعيد، ذلك أن التحليل التاريخي يجب أن ينصب في معظمه على الانتقال من حالة معقدة في الماضي إلى حالة أخرى معقدة في المستقبل، ولذا فإن منهجية التحليل التاريخي ذات أهمية جوهرية بالنسبة للعلوم الاجتماعية التي تتناج إلى هذه المنهجية من أجل تكهناةقا بالمستقبل.

أما الفصل الأخير الذي حمل عنوان نحو منهج إسلامي لإعادة كتابة التاريخ، فقد اشتمل على خلاصة رأي المؤلف في هذه المسالة التي شغلت أقلام العديد من الكتاب والمؤرخين في العقد الأخير من هذا القرن. وبيّن المؤلف أن مسوغات هذه الدعوة تنحصر في أن فصولاً عديدة من تاريخنا الإسلامي، كتبت بأقلام تحمل وجهة نظر استعمارية معادية، سيطرت عليها النزعة المحلية التي رفعت من شأن الوثنيات القديمة من فرعونية، وآشورية، وبابلية، وبربرية، وفينيقية، وغيرها.

ويخلص المؤلف إلى أن القرآن الكريم قدم لنا منهجاً متكاملاً للبحث التاريخي، تجاوز كل المثالب التي ابتليت بها المدارس التاريخية سواء الغربية منها أو الشرقية، فالقرآن الكريم في منهجه ينطلق من قاعدة توازن ما بين الروح والمادة، في ضوء تصور واضح للكون والإنسان، رابطاً ما بين المبدأ والمنتهى بحلقات الأنبياء، مؤكداً أهمية الحديث والسنة في كتابة التاريخ، وكل ذلك بأسلوب مختصر غاية الاختصار.

ملاحظات نقدية

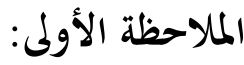

لقد حاول المؤلف -حفظه الله تعالى - في الفصل الأخير من كتابه أن يبيّن لنا أن القرآن الكريم، قدّم لنا أصول منهج متكامل للتعامل مع التاريخ، ولكننا لم نجد معالم هذا المنهج في تلك المعالجة، بل إن معالجة هذا الموضوع جاءت في منتهى الاختصار، علماً بأن هذا الموضوع يمثل عصب الكتاب، والنتيجة الأساسية التي من المفترض أن يقدمها، فلو توقف المؤلف عند هذا الموضوع وتعمّق في معالجته لكان ذلك أهم

$$
\text { إيجابيات تلك الدراسات وأهم نواحي تميزها. }
$$


وإسهاماً في إثراء هذا الموضوع سأحاول وبشكل مختصر تحديد أهم معالم المنهج وركائزه كما يقدمها القرآن الكريم للتعامل مع التاريخ وذلك تعميماً للفائدة، واستكمالاً للصورة التي قدمها الكتاب.

أجل، لقد قدّم لنا القرآن الكريم منهجاً متكاملاً للتعامل مع التاريخ، ذلك العلم الذي يمثل في رأي علماء الأمة أيام الله والمصدر الثالث من مصادر المعرفة الإنسانية.

إن من أهم ركائز هذا المنهج: التصور السليم والواضح للإنسان والكون والحياة، فالتصور هو الذي يهكم مناهج البحث ويعطيها الموازين التي تسير بموجبها، فالمناهج تعد أثراً من آثار التصور.

فالتصور القرآني يؤكد تناغم الإنسان مع الكون، فالخالق واحد، ومادة النشأة الأولى واحدة (الماء والتراب)، إلا أن الإنسان أكثر رفعةً من سائر مخلوقات الله في الكون، لذا سخّر الله الكون لهذا المخلوق. إن هذا التصور الذي قدمه القرآن الكريم يجعل المتمثل له أكثر وعياً واستيعاباً لحركة التاريخ، فهي حركة لا يحكمها الصراع بين الكون والإنسان، بل هو تاريخ يمثل في التصور الإسلامي سعي الإنسان لتحقيق ذاته في أبعادها كافة لتكن له الخلافة في الأرض في ظل هداية الوحي الإلهي. إنه تصور لا يتناقض مع سنن الكون وطبائع الأشياء، وييعل الإنسان هو مور التاريخ.

وتعدّ العقيدة الركيزة الثانية للمنهج الذي رسمه القرآن الكريم للتعامل مع التاريخ، فالعقيدة هي المركك الأساسي للتاريخ، فأحداث التاريخ منذ سيدنا آدم عليه السلام وحتى يرث الله الأرض ومن عليها، إنما هي قدر مقدور، ومن هنا كانت معرفة الماضي نافذة للإطلال على إرادة الله التي تمت في البشر، وكشفاً للمستقبل عن طريق هذا الماضي.

ويحتل مفهوم الشمولية ركناً مهماً في المنهج القرآي عند تعامله مع القضايا التاريخية، فالقرآن ينظر إلى الواقعة من مختلف زواياها، وفي إطارها الاجتماعي والاقتصادي، والسياسي، والجغرافي، والحضاري، والديني، والانفعالي، وحتى الجمالي بالنسبة للذين يصنعون الأحداث، لا بل إن شمولية النظرة لم قممل حتى الكوارث الطبيعة من سيول وفيضانات، وأمراض اجتماعية، وبالتالي ويع القرآن الكريم من أفق المؤرخ وجعله أكثر قدرة على التحليل والمقارنة والاستنتاج. 
إن هذا الفهم الشامل لحركة التاريخ الذي لا يعرف التجزئة للتجربة الإنسانية قد قدّمه القرآن الكريم في أجمل صورة من خلال القصص القرآني، وقد فهم مؤرخو الإسلام الإشارات القرآنية، فبدأوا مؤلفاقم التاريخية بالحديث عن سيدنا آدم عليه السلام إلى الوقت الذي يعيشون فيه، إيماناً منهم بوحدة التاريخ واستجابة لنداء القرآن الخالد الذي دعاهم لممارسة هذا الفهم. ولم يغب الزمن عن ركائز المنهج القرآني، فالقرآن الكريم يضع حداً فاصلاً بين زمانين على الأقل: الزمان الإلهي والزمان الإنساني. والزمان الإنساني نفسه مرتبط أوثق الارتباط بدرجة الوعي لدى الإنسان ومقدار حظه من المعرفة، وقدرته أخيراً على التذكر واستيعاب الأهداف والوقائع، وذلك ما توضح سورة الكهف؛ وتنوع مفهوم الزمن كما هو معلوم لم يتنبه له المؤرخون إلا في مرحلة متأخرة.

أما الأسلوب الذي يعد من الأدوات المهمة في المنهج فقد بيّن القرآن الكريم أن الأسلوب الأفضل في عرض الأحداث، هو الأسلوب الذي يرّز في العرض على الأهداف والغايات بأسلوب مختصر اختصاراً غير مخل وفي لغة يسهل فهمها وبتعلك تلمس الأحداث في حرارتا إبّان وقوعها وما يعقبها من صدى، أسلوب

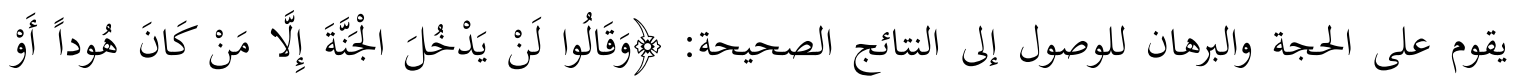

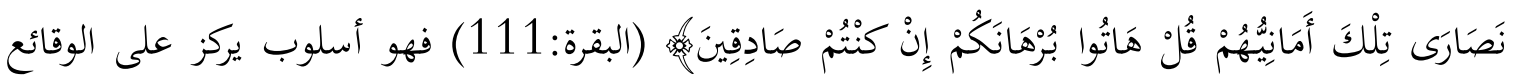

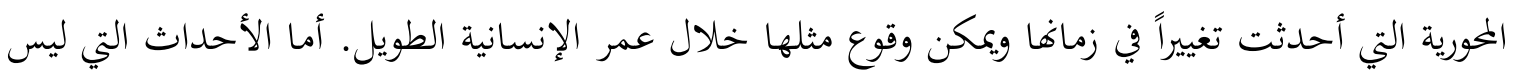
لها تأثير فتذكر بشكل بجمل، وكل ذلك بلغة تتميز بالدقة والسهولة والوضوح، والبعد عن التجريح الشخصي

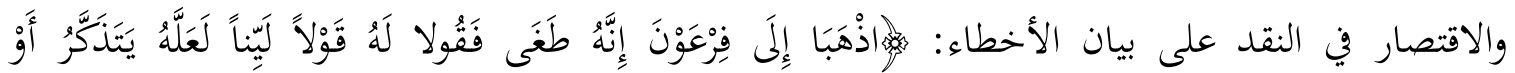
يَنْشَى كَهِ (طه:43-44).

ومن أركان المنهج القرآني (الموضوعية)، فقد شنَّ القرآن الكريم حملة شعواء على الهوى والظن والتعصب مركزاً في الوقت نفسه على الصدق والأمانة، وإظهار الشهادة وعدم إخفاء الحقائق، بل إن القرآن

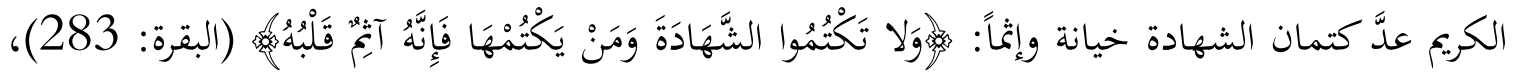

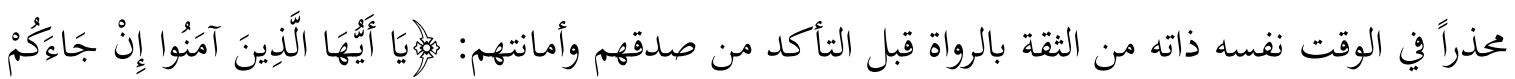

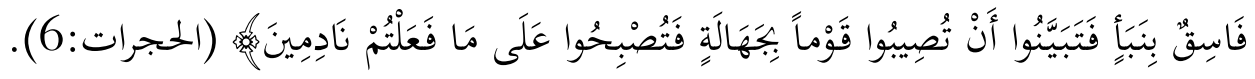


وقد أعطى القرآن الكريم للمصادر مساحة مهمة في المنهج الذي يدعو إليه، فقد وسّع من أفق المؤرخ وقدّم له تصوراً جديداً للمصادر عندما قسمها إلى عدة مجموعات تشمل كل أثر خلّفه الإنسان؛ ونظرة سريعة للإشارات القرآنية بتعلنا نقسمها إلى المجموعات الآتية: أ- الرواية الثفوية: وتبرز في المحاورات القرآنية، والروايات المنقولة على ألسنة الشخوص الذي ورد ذكرهم في القرآن.

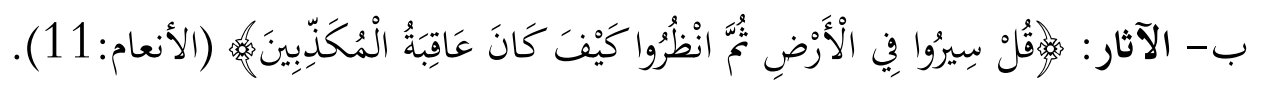
ج- المسكوكات: وهي من الأدلة المادية التي يعتمد عليها في التحقق من صحة المعلومات التاريخية، لذا كانت الإشارة القرآنية واضحة في سورة الكهف لهذا المصدر. د- الملابس: وتعد اليوم من المصادر المهمة التي تثبت العديد من الحقائق الدينية، والاجتماعية، والاقتصادية، والجمالية، وحتى السياسة، وقد جعل القرآن الكريم بإشارة خفية الملابس من وسائل الإثبات للحقائق في قصة سيدنا يوسف (عليه السلام) مع امرأة العزيز. هـ- الوثائق الرسمية: لقد اعتنى القرآن الكريم بهذا اللون من المصادر وهي تشتمل على الرسائل الرسمية، والوثائق المتعلقة بالمعاملات الاقتصادية، فقد أشار القرآن الكريم على سبيل المثال إلى رسالة سليمان (عليه السلام) إلى ملكة سبأ.

ويختم القرآن الكريم توجيهاته المنهجية في حقل المصادر بالإشارة إلى أن بعض المصادر قد يدخلها

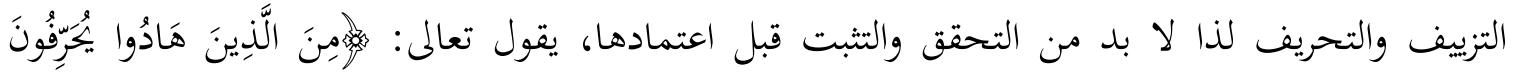

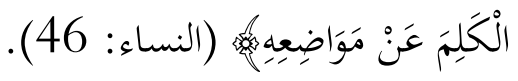

ويعطي القرآن الكريم للسببية أو العلية وزناً واضحاً في المنهج الذي يدعو إليه، وشرط السببية أن

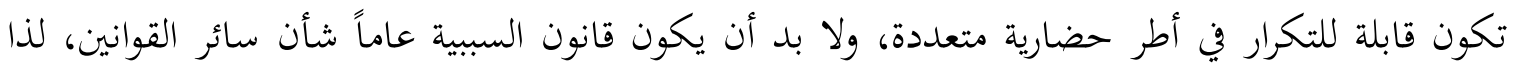
نجد القرآن الكريم عند حديثه عن عوامل سقوط الحضارات يركّز على عاملي الظلم والترف، ويقدّم هذه العلّة 
في عدّة صور عند أمم متعددة في عصور متباعدة ليقول لنا: إن العلّة تكتشف من خلال الاستقراء المتأني لمساحة كبيرة من الأحداث في عصور زمنية متباعدة وفي أماكن متباينة.

والركيزة الأخيرة في المنهج القرآني في التعامل مع التاريخ هي الواقعية، فهو منهج واقعي عملي، منهج

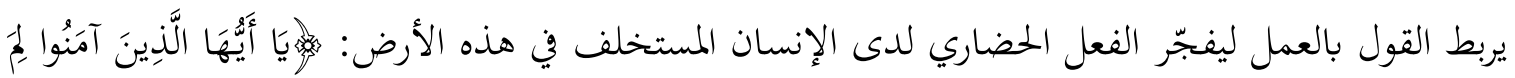

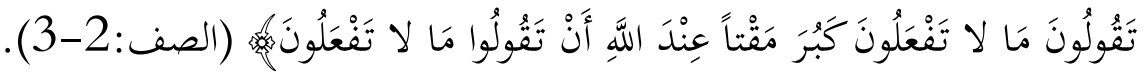
وهذا ما فهمه الشاطبي حين قال في مقدمة الجزء الأول من الموافقات: بأن كل مسألة لا يينى عليها عمل فالخوض فيها خوض فيما لم يدل على استحسانه دليل شرعي.

وهكذا يقدم لنا القرآن الكريم أصول منهج متكامل في التعامل مع وقائع التاريخ ومساراته. ونخن نتمنى على مؤلف الكتاب في حالة إعادة طبع كتابه أن يولي هذا الموضوع عناية خاصة لأنه قاعدة الانطلاق التي يجب أن ينطلق منها كل من يريد أن يؤصل للعلوم الإنسانية.

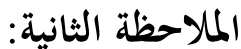

إن عدم تخصص المؤلف -حفظه الله تعالى- في الدراسات التاريخية جعله ييذل جهداً مضنياً مكرراً جهود من سبقه، فمن الملاحظ أن العديد من موضوعات الكتاب قد عولجت -على مسبيل المثال- بشكل مفصل في الكتب والبحوث الآتية التي تخلو منها قائمة مصادر ومراجع كتابه المسلمون وكتابة التاريخ وهي: 1- - محد بن صامل السلمي: منهج كتابة الثاريخ الإسلامي، الرياض، 1406هـ-

2- عبد الحليم عويس: تفسير التاريخ، علم إسلامي، القاهرة، 1407هـ |1987م. و"تفسير التاريخ في تراثنا الإسلامي"، الإسلام اليوم العدد الخامس. 3- محمد رشاد خليل: المنهج الإسلامي لدراسة التاريخ وتفسيره، الدار البيضاء: 
4- عثمان موافي: منهج النقد التاريخي الإسلامي والمنهج الأوروبي. الإسكندرية:

1984/ه1404

5- عماد الدين خليل: في التاريخ الإسلامي لماذا المنهج" الأمة، محرم، 1403هـ/

تشرين الثاني 1982م.

6- مصطفى الشكعة: "المنهج الإسلامي في فكر ابن خلدون في مواجهة دراسة الغربين". بحث مقدم إلى الندوة الدولية حول الدراسات والأبحاث في الحضارة الإسلامية، اسطنبول 15-18 صفر 1409هـ/26-29 سبتمبر 1988م.

7- محمد قطب: حول التفسير الإسلامي للتاريخ، الرياض، 1412هـ/1992م. 8 - محمود الشرقاوي: التفسير الديني للتاريخ، القاهرة، كتاب الشعب، بدون

إنَّ حضارتنا الإسلامية أنجبت أكثر من خمسة آلاف مؤرخ كتبوا في أربعين فرعاً من فروع التاريخ الإسلامي التي حدده الإمام الذهبي، ولك لون منها منهجه الخاص. فتأصيل المناهج يتطلب جهوداً مضنية تبذها مجموعات عمل متجانسة تعمل لتحقيق هذه الغاية. وفي اعتقادي أن الجهود الفردية تبقى محاولات مشكورة، ولكن من الصعب على المدى المنظور أن توصلنا إلى الغاية التي ننشدها. ورغم كل ذلك يبقى الكتاب كتاباً نافعاً للمثقف المسلم يعينه على تشكيل تصور عن أزمة المنهج التي تعاني منها الدراسات التاريخية في عالمنا الإسلامي. 